\title{
Diagnostic Value of Mean Elasticity Index as a Quantitative Shear Wave Elastography Parameter for Prediction of Malignancy in Small Suspicious Solid Thyroid Nodules
}

\author{
Saba Sohail and Sameera Kaliq uz Zaman \\ Department of Radiology, Dow Medical College, Dow University of Health Sciences and DRKP Civil Hospital, Karachi, Pakistan
}

\begin{abstract}
:
Objective: To determine the diagnostic accuracy of mean elasticity index $\left(E_{\text {mean }}\right)$ on quantitative shear wave elastography (SWE) in detection of malignancy in small solid suspected malignant thyroid nodules on ultrasound, taking histopathology as the gold standard.

Study Design: Analytical study.

Place and Duration of Study: Department of Radiology, Dow Medical College, Dow University of Health Sciences (DUHS) and Dr. Ruth K. M. Pfau Civil Hospital, Karachi from September 2017 to March 2018.

Methodology: Adult patients of either gender with suspected malignant thyroid nodules (solid, $\leq 10 \mathrm{~mm}$ ) were subjected to SWE examination. All patients underwent thyroid surgery later. Histopathological findings were used as the gold standard for determining sensitivity, specificity, positive predictive value (PPV), negative predictive value (NPV) and accuracy, at a cut-off $E_{\text {mean }}$ of 66 kilo pascals $(\mathrm{KPa})$. These values were further stratified for gender, age (below or above 40 years), single or multiple nodule(s), and known duration of nodules (more or less than 10 months).

Results: The average age of 109 women $(69.43 \%)$ and 48 men $(30.57 \%, n=157)$ was $37.64 \pm 9.44$ years. The mean nodule size was $6.83 \pm 3.16 \mathrm{~mm}$. The overall sensitivity, specificity, PPV, NPV and accuracy of the studied parameter in detection of malignancy was $81.5 \%, 92.3 \%, 68.8 \%, 96 \%$ and $90.5 \%$, respectively. Diagnostic accuracy was higher for females, age over 40 years, single nodule, and known duration for longer than 10 months.

Conclusion: $\mathrm{E}_{\text {mean }}$ - a quantitative SWE parameter - showed high diagnostic accuracy in predicting thyroid malignancy, particularly in older adults with sub-cm solid nodules of more than 10 months duration. Where available, SWE should be used to complement routine thyroid ultrasonography, which may be helpful to reduce unnecessary intervention.
\end{abstract}

Key Words: Mean elasticity index $\left(E_{\text {mean }}\right)$, Thyroid nodule, Malignancy, Shear wave elastography, Solid nodule.

How to cite this article: Sohail S, Zaman SKU. Diagnostic Value of Mean Elasticity Index as a Quantitative Shear Wave Elastography Parameter for Prediction of Malignancy in Small Suspicious Solid Thyroid Nodules. J Coll Physicians Surg Pak 2020; 30(07):683-687.

\section{INTRODUCTION}

Nodules in thyroid gland are frequently encountered in clinical practice. The reported estimate of frequency is $10 \%$ in women and $2 \%$ in men and less than $10 \%$ are malignant. ${ }^{1}$ Incidental detection of thyroid nodules can be as high as $68 \%$ on ultrasound (US), with only $5-15 \%$ proving malignant. ${ }^{2,3}$ In the Framingham study, the frequency of clinically apparent thyroid nodules was $6.4 \%$ in women and $1.5 \%$ in men. ${ }^{4}$

Correspondence to: Prof. Saba Sohail, Department of Radiology, Dow Medical College, Dow University of Health Science and DRKP Civil Hospital, Karachi,

Pakistan

E-mail: drsabasohail@hotmail.com

Received: June 21, 2020; Revised: July 04, 2020;

Accepted: July 06, 2020

DOI: https://doi.org/10.29271/jcpsp.2020.07.683
Whether detected by sight, palpation or imaging studies, US and fine-needle aspiration cytology (FNAC), play important roles in further evaluation of thyroid nodules, which is the characterisation into cystic versus solid and benign versus malignant nodules. ${ }^{5}$ While a completely cystic nodule is benign till it remains cystic, there are many US findings that are suggestive butnone is absolutely pathognomonic for thyroid cancer, particularly in its differentiation from a follicular adenoma. The reported sensitivity and specificity varies markedly at $55-90 \%$ and $52-81 \%$, respectively. ${ }^{6}$ While FNAC is considered to be the best and most common method for picking malignant nodules, it has its own limitations with a high proportion of indeterminate results (15-25\%) and again a widely varying sensitivity and specificity at $54-90 \%$ and $60-98 \%$, respectively. ${ }^{1,6}$

Stiffness of any tissue is due to its structural matrix, which is altered by pathology. Tumor has a closely packed cellularmatrix, which makes the lesion stiffer; this can be evaluated by elastog- 
raphy. Elastography is a relatively new imaging technique, which is still evolving. It has many versions and shear wave elastography (SWE), which evaluates the stiffness of a tissue in various ways, is a user-friendly version, rapidly gaining popularity. The stiffness is measured in many ways including strain ratios or color-coded grades or as elasticity index. ${ }^{7}$ A number of studies and reviews have established SWE as a very useful technique., ${ }^{7,8}$ Veyrieres et al. established a mean elasticity index $\left(\mathrm{E}_{\text {mean }}\right)$ value of 66 kilo pascals ( $\mathrm{KPa}$ ) as the best to determine malignancy. ${ }^{3}$ However, there are recent studies contradicting this differentiating ability. ${ }^{9}$

There are only two locally published studies available regarding SWE. One was conducted in Pakistan and evaluated strain ratios and scores. ${ }^{10}$ The other was conducted on Chinese population, which used color coding grades for determining the hardness of thyroid nodules. ${ }^{11}$ Due to lack of data on Pakistani population, we aimed to determine the diagnostic accuracy of a quantitative parameter in SWE, $E_{\text {mean }}$ for predicting malignancy is solid micronodules in thyroid gland suspected as malignant on B mode US taking histopathology as the gold standard.

\section{METHODOLOGY}

This analytical study was conducted at the Department of Radiology, Dow Medical College, Dow University of Health Sciences (DUHS) and Dr. Ruth K. M. Pfau Civil Hospital, Karachi from September 2017 to March 2018, started after the approval of synopsis from Research Evaluation Unit (REU), College of Physicians and Surgeons Pakistan (CPSP). Sample size was calculated on the basis of $15 \%$ prevalence of malignancy in all detected thyroid nodules in general population. ${ }^{3}$ Keeping prevalence at $15 \%$, sensitivity $80 \%$ (margin of error, 16.2\%), specificity $90.5 \%$ (margin of error, $05 \%$ ), desired precision of estimate $10 \%$, and confidence level at $95 \%$, sample size was calculated to be 157 .

Inclusion criteria were adults, 20-60 years of age, of either gender with solid sub-centimeter thyroid nodules suspected to be malignant on conventional US, with later surgical excision of the nodule and availability of the histopathology report. Exclusion criteria were patients with diffuse background thyroid lesions including Grave's disease and Hashimoto's thyroiditis, thyroid nodules on US occupying $>75 \%$ of thyroid lobe (as insufficient surrounding normal tissue will be available as normal reference in SWE), markedly calcified nodules hindering the placement of the $2 \times 2 \mathrm{~mm}$ region of interest (ROI) sample box, and complex nodules with both solid and cystic components. Those who did not undergo surgery or did not provide histopathology findings were also excluded.

Subjects fulfilling the inclusion criteria were enrolled; and written informed consent was obtained from all patients, prior to inclusion in the study. Patients with US showing suspicious solid thyroid micro-nodule underwent SWE examination using Aixplorer ${ }^{\mathrm{TM}}$ elastography machine with $15 \mathrm{MHz}$ linear transducer by a consultant radiologist having at least 3 years of experience in sonography, who was blinded to the patient identity and base- line US report. $\mathrm{E}_{\text {mean }}$ was determined by placing a $2 \times 2 \mathrm{~mm}$ sample box away from the margin of the nodule showing the most homogeneity without calcifications or vessels. Mean elasticity of the normal intervening tissue was checked for reference. Elastography findings were entered in a customised proforma. All patients werefollowed for surgery and biopsy reportthroughtelephonic communication. Histopathological findings of each patient were inquired from the consultant histopathologist on telephone or through written report and endorsed.

Diagnostic accuracy was evaluated in terms of sensitivity, specificity, positive predictive value (PPV) and negative predictive value (NPV) by taking histopathology as reference standard.

Elasticity index (EI) is the quantitative measurement of tissue stiffness by system software by placing the built-in ROI box $(2 \times 2 \mathrm{~mm})$ over the stiffest part of the nodule that is expressed in $\mathrm{kPa}$ as $\mathrm{E}_{\text {mean }}$ (mean value for the ROI placed in the stiffest region), $E_{\max }$ and $E_{\min }$. Malignant nodule on SWE was defined as the lesion based on the $\mathrm{El}$ with $\mathrm{E}_{\text {mean }}$ value $>66 \mathrm{kPa}$. Benign nodule on SWE was defined as the lesion based on the EI with $E_{\text {mean }}$ value $\leq 66 \mathrm{kPa}$. Malignant nodule criteria on B mode US included varying combination of micro-calcification, intranodular vascularity, irregular or micro-lobulated margins, taller than wider dimensions, and absence of halo sign (absence of anechoic/hypoechoic rim around a nodule).

True positive (TP) was a thyroid nodule declared malignant on SWE as well as on histopathology; true negative (TN) was a nodule labelled benign on SWE as well as on histopathology. False positive (FP) was a thyroid nodule benign on histopathology but labelled malignant on SWE; false negative (FN) was a thyroid nodule malignant on histopathology but benign on SWE.

Data was evaluated and analysed using statistical programme for social sciences (SPSS) version 13.0. Mean, standard deviation (SD), median and interquartile range (IQR) were calculated for continuous variables (age, El, duration of swelling, size of nodules), while frequency and percentage were calculated for nominal/ordinal data (gender, number of thyroid nodules (single/multiple). Discriminating potential of the SWE was evaluated using the standard characteristics of diagnostic evaluation, i.e. sensitivity, specificity, PPV and NPV using histopathology as the gold standard and constructing $2 \times 2$ tables. Stratification was done for age as under and over 40 years, gender, known duration of nodules (as thyroid lumps) for less or more than 10 months, and number of thyroid nodules (single or multiple) to see the effect of these on diagnostic accuracy.

\section{RESULTS}

A total of 157 patients meeting the selection criteria were inducted. The average age was $37.64 \pm 9.44$ years. The mean duration of thyroid lumps, size of nodules and SWE measurement, i.e., $E_{\text {mean }}$ are shown in Table I. There were 48 (30.57\%) males and 109 (69.43\%) females. One hundred and forty-three $(91.08 \%)$ patients had single nodule and 14 (8.92\%) had multiple nodules. 
Table I: Characteristics of patients with thyroid nodules.

\begin{tabular}{|c|c|c|c|c|c|c|}
\hline \multirow{2}{*}{ Variables } & \multirow{2}{*}{ Mean } & \multirow{2}{*}{ Std. Deviation } & \multicolumn{2}{|c|}{ 95\% Confidence interval for mean } & \multirow{2}{*}{ Median } & \multirow{2}{*}{ IQR } \\
\hline & & & Lower bound & Upper bound & & \\
\hline Age (years) & 37.64 & 9.44 & 36.15 & 39.13 & 36 & 11 \\
\hline Duration of swelling (months) & 16.94 & 13.21 & 14.86 & 19.03 & 10 & 23 \\
\hline Size of nodule $(\mathrm{mm})$ & 6.83 & 3.16 & 6.34 & 7.33 & 6 & 3 \\
\hline $\mathrm{E}_{\text {mean }}(\mathrm{KPa})$ & 53.07 & 13.08 & 51.01 & 55.13 & 55 & 19 \\
\hline
\end{tabular}

Table II: Overall and group-wise break-up of accuracy, sensitivity, specificity, and predictive values.

\begin{tabular}{|c|c|c|c|c|c|c|}
\hline Parameter & No. & Sensitivity \% & Specificity \% & PPV \% & NPV \% & Accuracy \% \\
\hline Over all & $157(100 \%)$ & 81.5 & 92.3 & 68.8 & 96 & 90.5 \\
\hline \multicolumn{7}{|l|}{ Age (years) } \\
\hline Under 40 & $108(68.79 \%)$ & 77.3 & 89.5 & 65.4 & 93.9 & 87 \\
\hline Over 40 & $49(31.21 \%)$ & 100 & 97.7 & 83.3 & 100 & 98 \\
\hline \multicolumn{7}{|l|}{ Gender } \\
\hline Male & $48(30.57 \%)$ & 100 & 75 & 66.7 & 100 & 83.3 \\
\hline Female & $109(69.43 \%)$ & 54.6 & 98 & 75 & 95.1 & 93.6 \\
\hline \multicolumn{7}{|l|}{ Number of nodules } \\
\hline Single & $143(91.08 \%)$ & 70.6 & 95.2 & 66.7 & 96 & 92.3 \\
\hline Multiple & $14(8.92 \%)$ & 100 & 00 & 71.4 & 00 & 71.4 \\
\hline \multicolumn{7}{|c|}{ Known duration of nodule } \\
\hline Less than 10 months & $85(54.14 \%)$ & 80 & 86.2 & 64 & 93.3 & 84.7 \\
\hline 10 months or more & $72(45.86 \%)$ & 85.7 & 98.5 & 85.7 & 98.5 & 97.2 \\
\hline
\end{tabular}

Overall SWE predicted malignancy in $32(20.38 \%)$ nodules and excluded it in 125 (79.62\%) labelling them as benign. The surgeons proceeded to excise based on the suspicion raised by the US report. Histopathology confirmed 27 (17.2\%) malignant nodules and found 130 (82.8\%) benign nodules such that there were 22 TPs, 120 TNs, 10 FPs and 05 FN results. Sensitivity, specificity, PPV, NPV and accuracy of SWE in detection of malignancy in suspected malignant thyroid nodule were $81.5 \%, 92.3 \%, 68.8 \%, 96 \%$ and $90.5 \%$, respectively. Accuracy according to age stratification, was higher for those over 40 years of age as shown in Table II. Diagnostic accuracy was $83.3 \%$ and $93.6 \%$ for males and females, respectively. Diagnostic accuracy of SWE in detection of malignancy in suspected malignant thyroid nodule was $92.3 \%$ for single nodules and $71.4 \%$ for multiple nodules (Table II). A markedly higher accuracy was found for single compared to multiple nodules (Table II).

\section{DISCUSSION}

Detection of small thyroid nodules has increased in recent years with widespread use of imaging modalities. ${ }^{12}$ Determination of the nature of the nodules as benign or malignant is the foundation for its subsequent treatment. ${ }^{13}$ US and FNAC are commonly employed for this purpose but both have some limitations. Hence, there is a quest for new modalities and parameters for supplementing these techniques avoiding invasive procedures for the benign nodules, which are much more numerous than the malignant nodules. The present study evaluated the diagnostic potential of a quantitative parameter on SWE for this purpose and found it highly accurate and minimally fallacious.

Elastography has evolved during the last decade as a noninvasive and easily available diagnostic imaging modality for the evaluation of thyroid nodules. ${ }^{14}$ It is a promising technology based on measuring the tissue strain, well suited for thyroid gland due to its anatomical positiong. ${ }^{15}$ SWE measures the shear wave velocity of the tissue by acoustic radiation force impulse; and the velocity reflects the tissue hardness directly with Young modulus. With the image of shear wave propagation velocity through tissues, qualitative and quantitative assessments about the surrounding tissues are performed. These may be expressed in various ways as scores, grades, strain ratios and elasticity index. ${ }^{7}$ In this study, $E_{\text {mean }}$ was employed to determine the diagnostic accuracy of quantitative SWE in detection of malignancy in suspected malignant thyroid nodules on US with a cut off value hailed as being the best in a previous study. ${ }^{3}$ 
In recent years, extensive studies about the diagnostic role of SWE in thyroid nodules have been conducted. Samir et al. concluded that SWE showed superiority in the diagnosis of malignant thyroid nodules compared to conventional US. ${ }^{16}$ However, contrary views also abound such as those expressed by Swan et al. and Bhatia et al. ${ }^{9,17}$ This might be due to differences in the size of the nodules, various parameters applied, and multiplicity of nodules as found by Swan et al. in their regression analysis. ${ }^{9}$

Our results reflected high diagnostic accuracy of SWE in malignant thyroid nodules. These results confirmed the results from a previous meta-analysis by Zhang et al. that also reported that SWE had high sensitivity and specificity in evaluating thyroid nodules. ${ }^{2}$ A similar conclusion was drawn by Lin et al. in their meta-analysis on this topic. ${ }^{18}$ The present results were also comparable to the study by Veyrieres et al. which had established the cutoff parameter as used in this study i.e., $66 \mathrm{KPa}$. $^{3}$ They found a sensitivity of $80 \%$ and specificity of $90.5 \% .^{3}$ On the other hand, Swan et al. maintained that there is a big overlap of the $E_{\text {mean }}$ in benign and malignant nodules. ${ }^{9}$ The overall lower values of $E_{\text {mean }}$ in this study are most likely due to the preponderance of benign nodules.

The study by Nazir et al. used strain ratio as the parameter for SWE in the index local study finding $81.82 \%$ sensitivity, 95.45\% specificity, 56.25\% PPV, 98.65\% NPV and $98.09 \%$ accuracy. Their population was nearly of the same age (35.81 \pm 9.03 years) as the presently studied population (37.64 \pm 9.44 years). However, the frequency of malignant nodules in their study was much lower with only $6.67 \%$ of all nodules turning out to be malignant. That might be due to using FNAC and not histopathology of the excised nodules as the reference for comparison.

Wong et al. studied surgically excised micro-nodules as done in the present study. Using color coding grades for the nodules, they found a sensitivity of $88.9 \%$ and specificity of $89.3 \% .{ }^{11}$ The diagnostic accordance rates were comparable to conventional US. Their subjects were older (mean age $43.4 \pm 10.8$ years) but there was no mention of the exact relative proportion of the benign and malignant nodules.

These results were further stratified for age, gender, multi plicity and duration of nodules. An overall higher diagnostic accuracy was found for females, older age, single nodules and longer duration of nodules. There were $30.57 \%$ men and $69.43 \%$ women in this study showing female prevalence in thyroid disorders consistent with majority of previous studies. ${ }^{6,10,11}$ of particular note is the low specificity and high NPV for males on subgroup analysis. This is an interesting finding and may be due to lower proportion of males having malignant nodules. Likewise, the zero specificity and NPV for multiple nodules may be due to their smaller proportion or because of sample selection from the number of nodules.
Swan et al. also mentioned the multiplicity of nodules as one of the factors related to the suboptimal performance of SWE. ${ }^{9}$

The limitations of this study include studying only micronodules and not large nodules. There might be sampling errors in case of multiple nodules. However, this is the first Pakistani study that has studied SWE in thyroid micronodules. The stratification of accuracy results according to age, gender and duration of thyroid nodules are a novel addition to the existing relevant literature. Although the number of FP and FN cases was low, yet the authors recommend using SWE in conjunction with the B-mode US to further improve the diagnostic performance and not as the sole imaging, as recommended by others. ${ }^{19,20}$

\section{CONCLUSION}

$\mathrm{E}_{\text {mean }}$ a quantitative SWE parameter, showed high diagnostic accuracy in predicting thyroid malignancy, particularly in older adults with sub-cm solid nodules of more than 10 months duration. Where available, SWE should be used to complement routine thyroid ultrasonography, which may be helpful to reduce unnecessary intervention.

\section{DISCLOSURE:}

This article is based on the dissertation written by SK in partial fulfilment of the requirements of FCPS in Diagnostic Radiology, approved vide: CPSP/REU/RAD-2014-183-1745.

\section{ETHICAL APPROVAL:}

Synopsis approved from CPSP prior to the study.

\section{CONFLICT OF INTEREST:}

First author is one of the editors of JCPSP. The manuscript was processed through other editors.

\section{AUTHORS' CONTRIBUTION:}

SS: Conceived the project, critically reviewed the draft of dissertation, comprehensively and substantively rewrote as article, and updated the references.

SKZ: Collected and analysed the data, searched literature, and wrote synopsis and dissertation.

\section{REFERENCES}

1. Islam N, Ahmed A. Management of thyroid nodules: An optimal approach. J Col Physicians Surg Pak 2011; 21(8): 447-9.

2. Zhang B, Ma X, Wu N, Liu L, Liu X, Zhang J, et al. Shear wave elastography for differentiation of benign and malignant thyroid nodules- a meta-analysis. J Ultrasound Med 2013; 32(12):2163-9.

3. Veyrieres JB, Albareld F, Vaillant LJ, Berbise J, Sebag F, Oliver $C$, et al. A threshold value in shear wave elastography to rule out malignant thyroid nodules: A reality? J Euro Rad 2012; 81(12):3965-72.

4. Vander JB, Gaston EA, Dawber TR. The significance of 
nontoxic thyroid nodules. Final report of a 15-year study of the incidence of thyroid malignancy. Ann Intern Med 1968; 69(3):537-40

5. Hu X, Liu Y, Qian L. Diagnostic potential of real-time elastography (RTE) and shear eave elastography (SWE) to differentiate benign and malignant thyroid nodule. Medicine 2017; 96(43):e8282.

6. Tian W, Hao S, Gao B, Jiang Y, Zhang S, Guo L, et al. Comparison of diagnostic accuracy of real-time elastography and shear wave elastography in differentiation malignant from benign thyroid nodules. Medicine 2015; 94(52): e2312.

7. Zhao $\mathrm{C}, \mathrm{Xu} \mathrm{H}$. Ultrasound elastography of the thyroid: Principles and current status. Ultrasonography 2019; 38(2): 106-24.

8. Huang R, Jiang L, Xu Y, Gong Y, Ran H, Wang Z, et al. Comparative diagnostic accuracy of contrast-enhanced ultrasound and shear wave elastography in differentiating benign and malignant lesions; A network meta-analysis. Front Oncol 2019; 9:102.

9. Swan KZ, Bonnema SJ, Jespersen ML, Nielsen VE. Reappraisal of shear wave elastography as a diagnostic tool for identifying thyroid carcinoma. Endocrine Connections 2029; 8(8):1195-1205.

10. Nazir M, Imran M, Mirza TM, Tariq A. Role of elastography in differentiating malignant and benign thyroid nodules. Pak J Radiol 2017; 27:200-3.

11. Wong J, Wei W, Guo R. Ultrasonic elastography and conventional ultrasound in the diagnosis of thyroid micronodules. PakJ Med Sci 2019; 35(6):1526-31.

12. Knox MA. Thyroid nodules. Am Fam Physician 2013; 88:193-6.
13. Hegedus L, Bonnema SJ, Bennedbaek FN. Management of simple nodular goiter: Current status and future perspectives. Endocr Rev 2003; 24(1):102-32.

14. Hegedus L. Can elastography stretch our understanding of thyroid histomorphology? J Clin Endocrinol Metab 2010; 95(12):5213-5.

15. Kim JY, Lee $\mathrm{CH}$, Kim SY. Radiologic and pathologic findings of non-palpable thyroid carcinomas detected by ultrasonography in a medical screening center. J Ultrasound Med 2008; 27(2):215-23.

16. Samir AE, Dhyani M, Anvari A. Shear-wave elastography for the preoperative risk stratification of follicular-patterned lesions of the thyroid: Diagnostic accuracy and optimal measurement plane. Radiology 2015; 277(2):565-73.

17. Bhatia KS, Tong CS, Cho CC. Shear wave elastography of thyroid nodules in routine clinical practice: Preliminary observations and utility for detecting malignancy. Eur Radiol 2012; 22(11):2397-406.

18. Lin $\mathrm{P}$, Chen M, Liu B. Diagnostic performance of shear wave elastography in the identification of malignant thyroid nodules: A meta-analysis. Eur Radiol 2014; 24(11): 2729-38.

19. Han R, Du J, Li F, Zong H, Wang J, Shen $Y$, et al. Comparisons and combined application of two-diemensional and three-dimensional real-time shear wave elastography in diagnosis of thyroid nodule. J Cancer 2019; 10(9):1975-84.

20. Yeon EK, Sohn Y, Seo M, Kim E, Eun Y, Park WS, et al. Diagnostic performance of a combination shear wave elastography and B-mode ultrasonography in differentiating benign from malignant thyroid nodules. Clin Exp Otorhinolarnygol 2019; 12(2):186-193. 